\title{
Assessment of image quality and low-contrast detectability in abdominal CT of obese patients: comparison of a novel integrated circuit with a conventional discrete circuit detector at different tube voltages
}

\author{
A. Euler • T. Heye $\cdot$ M. Kekelidze $\cdot$ G. Bongartz • \\ Z. Szucs-Farkas • C. Sommer • B. Schmidt • \\ Sebastian T. Schindera
}

Received: 28 April 2014 /Revised: 10 September 2014 / Accepted: 29 September 2014 /Published online: 15 October 2014

(C) European Society of Radiology 2014

\begin{abstract}
Objectives To compare image quality and low-contrast detectability of an integrated circuit (IC) detector in abdominal CT of obese patients with conventional detector technology at low tube voltages.

Methods A liver phantom with 45 lesions was placed in a water container to mimic an obese patient and examined on two different CT systems at 80,100 and $120 \mathrm{kVp}$. The systems were equipped with either the IC or conventional detector. Image noise was measured, and the contrast-to-noise-ratio (CNR) was calculated. Low-contrast detectability was assessed independently by three radiologists. Radiation dose was estimated by the volume $\mathrm{CT}$ dose index $\left(\mathrm{CTDI}_{\mathrm{vol}}\right)$.

Results The image noise was significantly lower, and the CNR was significantly higher with the IC detector at 80 , 100 and $120 \mathrm{kVp}$, respectively $(P=0.023)$. The IC detector resulted in an increased lesion detection rate at $80 \mathrm{kVp}$ ( $38.1 \%$ vs. $17.2 \%)$ and $100 \mathrm{kVp}(57.0 \%$ vs. $41.0 \%)$. There was no difference in the detection rate between the IC detector at $100 \mathrm{kVp}$ and the conventional detector at $120 \mathrm{kVp}(57.0 \%$
\end{abstract}

A. Euler $\cdot$ T. Heye $\cdot$ M. Kekelidze $\cdot$ G. Bongartz $\cdot$

S. T. Schindera $(\bowtie)$

Clinic of Radiology and Nuclear Medicine, University of Basel

Hospital, Petersgraben 4, 4031 Basel, Switzerland

e-mail: sschindera@aol.com

Z. Szucs-Farkas

Institute of Radiology, Hospital Centre of Biel, Biel, Switzerland

C. Sommer

Department of Diagnostic and Interventional Radiology, University

Hospital, Heidelberg, Germany

B. Schmidt

Siemens Healthcare Sector, Forchheim, Germany vs. $62.2 \%$ ). The $\mathrm{CTDI}_{\mathrm{vol}}$ at 80,100 and $120 \mathrm{kVp}$ measured 4.5-5.2, 7.3-7.9 and 9.8-10.2 mGy, respectively.

Conclusions The IC detector at $100 \mathrm{kVp}$ resulted in similar low-contrast detectability compared to the conventional detector with a $120-\mathrm{kVp}$ protocol at a radiation dose reduction of $37 \%$.

Key Points

- An integrated circuit (IC) detector significantly improves the quantitative image quality

- The IC detector results in a significant improvement of lowcontrast detectability

- Reduction of tube voltage to $100 \mathrm{kVp}$ is feasible in obese patients

- The IC detector holds great promise for improving patient safety

Keywords Abdominal CT · Obesity · Radiation dose reduction $\cdot \mathrm{CT}$ detector $\cdot$ Diagnostic efficacy

\section{Introduction}

In the last two decades, the prevalence of obesity has increased throughout most industrialized countries [1], especially childhood obesity, which rose dramatically during the last 20 years [2]. Consequently, an even higher obesity rate is expected in the future. Obesity is a major risk factor for lifethreatening diseases associated with metabolic syndrome, such as stroke, myocardial infarction [3] and several types of cancer (e.g. colon cancer, postmenopausal breast cancer, endometrial cancer) $[4,5]$. Because computed tomography (CT) plays a key role in the diagnosis and follow-up of these (co-)morbidities, a rising number of future CT examinations should be expected. 
Two major problems of CT imaging in obese patients are the degraded image quality due to high image noise and artefacts (e.g. beam hardening and photon starvation artefacts) and the significantly higher radiation doses compared to average-sized patients $[6,7]$. While the risk of missing subtle pathologies exists, these patients acquire a higher cumulative lifetime radiation exposure, which carries an increased risk for developing radiation-induced cancer [8, 9]. In addition, recent scientific publications have established a correlation between a CT examination and the small but increased cancer risk in children and young adults $[10,11]$. Thus, every effort is required to optimize the radiation dose and image quality in obese patients undergoing a $\mathrm{CT}$ examination.

In addition to the iterative reconstruction technique, developmental refinement in detector technology offers a strategy to reduce radiation dose in obese patients while maintaining constant image quality. A recently introduced integrated circuit (IC) detector has the potential to reduce image noise by reducing the loss of information during the analogue-to-digital signal conversion in comparison to conventional, discrete circuit (DC) detector technology [12]. In particular, obese patients might benefit from an improved diagnostic performance at lower radiation doses by applying this novel development. However, to the best of our knowledge no data on the image quality and diagnostic performance is available for the IC detector in obese patients undergoing $\mathrm{CT}$ at different tube voltages. This prompted us to design a phantom with subtle simulated pathologies that allows for multiple, repeatable CT studies with different technical parameters. CT phantoms have the benefit of being robust and objective because no separate reference standard is required.

Thus, the purpose of our phantom study was to assess the image quality and low-contrast detectability of this novel IC detector in abdominal CT of obese patients compared with conventional DC technology at different tube voltages.

\section{Materials and methods}

\section{Phantom}

A custom liver phantom (QRM, Moehrendorf, Germany) was designed to mimic the liver parenchyma during the portal venous phase. The liver phantom has a cylindrical shape and contains 45 hypodense spherical lesions that simulate hypodense liver tumours. The liver parenchyma and the lesions are composed of a homogenous mixture of resin, including additives such as calcium carbonate and organic iodine. Thus, their Hounsfield units follow the pattern of the in vivo organ at various CT tube voltages. The lesions have three different diameters $(5,10$ and $15 \mathrm{~mm})$ and three different lesion-to-liver contrast values $(10,25$ and $50 \mathrm{HU}$ at $120 \mathrm{kVp}$ ). For each size and lesion-to-liver contrast value, there are five different lesions that are intentionally distributed throughout the phantom to create axial CT slices with multiple lesions, one lesion or without lesions. The construction plan was used as the reference standard for lesion location. The liver phantom was placed in a water-filled cylindrical plastic container with a diameter of $40 \mathrm{~cm}$, mimicking the abdominal cross-sectional dimension of an obese patient with an estimated body weight range of 118-142 kg (260-313 lbs) (Fig. 1) [13].

\section{CT scanning}

The liver phantom was scanned using two different 128 section multidetector CT systems (both SOMATOM Definition Flash, Siemens, Forchheim, Germany), one equipped with the conventional DC detector and the other equipped with the novel IC detector (Stellar detector, Siemens). The technical parameters for the CT protocols and the image reconstruction technique were identical for each $\mathrm{CT}$ system. Tube voltages of 80,100 and $120 \mathrm{kVp}$ were applied in combination with the use of tube current modulation software (CAREDose4D; Siemens Healthcare). Thus, a total of six CT data sets were available for further assessment. The $120-\mathrm{kVp}$ is the standard single-energy abdominal CT protocol recommended by the CT manufacturer. In addition, the following CT parameters were used: $150 \mathrm{mAs}$ quality reference tube

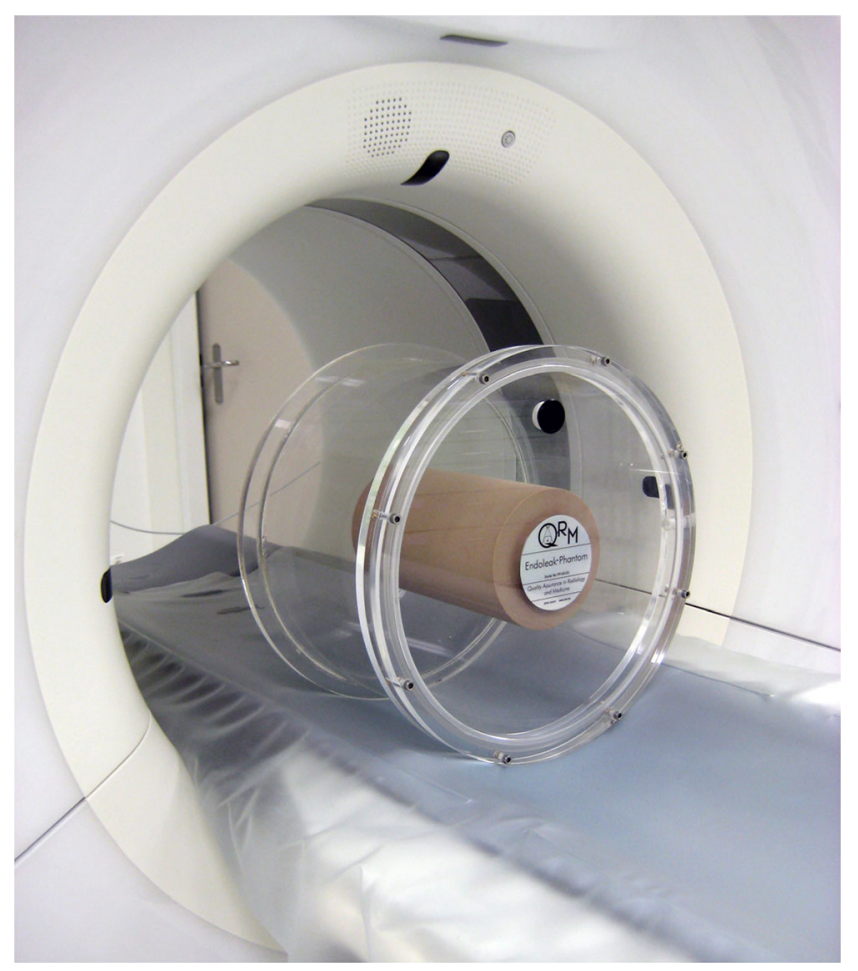

Fig. 1 Custom liver phantom placed in a water-filled cylindrical plastic container mimicking an obese patient. The liver phantom contains 45 hypoattenuating lesions with three different diameters $(5,10$ and $15 \mathrm{~mm})$ and three different lesion-to-liver contrast values (10, 25 and $50 \mathrm{HU})$ 
current-time product, $64 \times 0.6-\mathrm{mm}$ collimation, pitch of 0.6 and gantry rotation speed of $0.5 \mathrm{~s}$. The data sets were reconstructed in 5-mm axial images using the iterative reconstruction technique (SAFIRE, Siemens) and a reconstruction kernel of I30f. A strength of three was applied for the iterative reconstruction, as recommended by the manufacturer.

\section{Integrated circuit detector}

Conventional, discrete CT detector systems consist of a physical unit and an analogue-to-digital converter (ADC), which are located on two separate boards. The physical unit contains solid-state ceramic scintillators that convert x-rays into light, which is then converted into an electric signal. In the next step, this analogue signal is further translated and digitized by an ADC, which is located separately on an external board. This physical distance is a potential source of electronic noise. The recently introduced IC detector (Stellar detector, Siemens) aims to reduce this physical distance by attaching the physical unit and the ADC to the same board, potentially reducing electronic noise, power consumption and heat dissipation [12]. According to the manufacturer's instructions, the highest impact of noise reduction can be expected with the IC detector at low tube voltages such as $80 \mathrm{kVp}$.

\section{Radiation dose assessment}

For radiation dose assessment, the volume CT dose index $\left(\mathrm{CTDI}_{\mathrm{vol}}\right)$ was recorded for images from the dose page provided by the $\mathrm{CT}$. We did not calculate size-specific dose estimates [14] because the phantom diameter was constant.

\section{Assessment of objective image quality}

The Hounsfield units of the liver parenchyma and the surrounding water were measured by one of the authors, who is a second-year radiology resident (A.E.), by placing circular regions of interest (ROI). The size of the ROIs was $35 \mathrm{~cm}^{2}$ for both the liver parenchyma and the surrounding water. All measurements were acquired three times, and a mean value was calculated. The standard deviation of the Hounsfield units of the liver parenchyma served as image noise. Contrast-tonoise ratio (CNR) was calculated as (ROI (l)-ROI (w)) $/ N$ [ROI (l) = mean Hounsfield units of the liver parenchyma; ROI $(w)=$ mean Hounsfield units of the surrounding water; $N=$ mean image noise $]$.

Assessment of low-contrast lesion detection and subjective image quality

Three board-certified radiologists (G.B., T.H., M.K.) with 27, 8 and 4 years of experience in abdominal imaging analysed the six CT data sets $(80,100$ and $120 \mathrm{kVp}$ with DC and IC detector) independently on a high-definition liquid crystal display monitor with a preset soft-tissue window for abdominal imaging (width $400 \mathrm{HU}$, level $40 \mathrm{HU}$ ). The readers were free to change the window width and level for analysis. They were blinded to the number, position and diameter of the lesions in addition to which detector was used and the tube voltage. There were 19 axial images (5-mm thick) with one to five simulated lesions and 32 images with no lesions. The readers were asked to assess the diameter, position and grade of conspicuity of every lesion on evaluation sheets. The confidence was graduated in the following three categories: $1=$ maybe present; $2=$ most likely present; $3=$ definitely present. Each reader also evaluated subjective image noise (grade $1=$ major, unacceptable; grade $2=$ substantial, above average; grade $3=$ moderate, average; grade $4=$ minor, below average; grade $5=$ absent) and image quality (grade $1=$ bad, no diagnosis possible; grade $2=$ poor, diagnostic confidence substantially reduced; grade $3=$ moderate, but sufficient for diagnosis; grade $4=$ good; grade $5=$ excellent) for each data set on a 5 point scale. The six CT data sets were reviewed in six separate reading sessions, separated by a minimum of 1 week, in the following order: (a) DC detector at $80 \mathrm{kVp}$, (b) IC detector at $80 \mathrm{kVp}$, (c) DC detector at $100 \mathrm{kVp}$, (d) IC detector at $100 \mathrm{kVp}$, (e) DC detector at $120 \mathrm{kVp}$ and (f) IC detector at $120 \mathrm{kVp}$. The order of the reading was chosen to start with the data set demonstrating the most difficult task and ending with the data set representing the easiest task.

\section{Statistical analysis}

Marks made by readers in all six data sets were compared with the real localization of the lesions according to the construction plan of the liver phantom. True-positive and false-positive ratings by the three readers were analysed using the jackknife alternative free-response receiver operational characteristic (JAFROC) as described by Chakraborty [15]. This method is capable of analysing multiple findings per image including localization information, rewarding correct marks and penalizing incorrect marks made by the readers. Performance with each $\mathrm{CT}$ data set was characterized by the area under the JAFROC curve (AUC). Comparison of CT data sets included calculation of the $95 \%$ confidence intervals (CI) for the differences between AUCs. The probability of detection for each simulated lesion on the different $\mathrm{CT}$ series was pairwise compared using the Wilcoxon matched pairs test. Analysis of variance with post hoc tests was used to compare the objective quality data and subjective quality ratings were compared by non-parametric analysis of variance. All tests other than JAFROC were done using the Statistica software (Statsoft, Tulsa, OK). $P$ values of less than 0.05 were considered as significant. 


\section{Results}

Radiation dose

The radiation dose between the two CT systems equipped with different detectors was comparable within each tube voltage selection (Table 1). The radiation dose decreased up to $36 \%$ at $100 \mathrm{kVp}$ and up to $63 \%$ at $80 \mathrm{kVp}$ compared to $120 \mathrm{kVp}$. The application of the automatic tube current modulation resulted in a slight increase of the effective tube current-time product while decreasing the tube voltage from 120 to $80 \mathrm{kVp}$ (Table 1).

\section{Objective image quality}

The IC detector resulted in a substantial image noise reduction and CNR increase compared with the DC detector at the same tube voltage (Table 1). The image noise was 37.6, 15.0 and $8.3 \%$ lower and the CNR was 90.9, 15.3 and $10.9 \%$ higher for the IC compared with the DC detector at 80, 100 and $120 \mathrm{kVp}$, respectively $(P=0.023)$. The image noise was $25.7 \%$ higher, and the CNR was $6.8 \%$ lower with the IC detector at $100 \mathrm{kVp}$ compared with the DC detector at $120 \mathrm{kVp}(P=0.0001$ and 0.01 , respectively).

Low-contrast lesion detection and subjective image quality

The detection rate of the hypodense liver lesions was higher using the IC detector compared with the conventional detector at $80 \mathrm{kVp}(38.1 \%$ vs. $17.2 \%)$ and $100 \mathrm{kVp}(57.0 \%$ vs. $41.0 \%$ ) (Table 2). Similar results were seen for the lesion detection rate for tube voltage of $120 \mathrm{kVp}(62.2 \% \mathrm{vs} 60.7 \%)$. At 80 and $100 \mathrm{kVp}$, there was also a tendency for higher detection rates for lesions with a smaller diameter and a lower

Table 1 Data for effective tube current-time product, radiation dose and objective image quality

\begin{tabular}{lllll}
\hline $\begin{array}{l}\text { Tube voltage and } \\
\text { detector type }\end{array}$ & $\begin{array}{l}\text { Reference } \\
\text { mAs/effective mAs }\end{array}$ & $\begin{array}{l}\mathrm{CTDI}_{\mathrm{vol}} \\
(\mathrm{mGy})\end{array}$ & $\begin{array}{l}\text { Image } \\
\text { noise (HU) }\end{array}$ & $\mathrm{CNR}$ \\
\hline $80 \mathrm{kVp}$ & & & & \\
$\mathrm{DC}$ & $150 / 310$ & 6.1 & $51.3 \pm 2.5$ & $2.2 \pm 0.1$ \\
$\mathrm{IC}$ & $150 / 300$ & 5.9 & $32.0 \pm 2.6$ & $4.2 \pm 0.3$ \\
$100 \mathrm{kVp}$ & & & & \\
DC & $150 / 257$ & 10.6 & $24.7 \pm 1.5$ & $5.9 \pm 0.3$ \\
IC & $150 / 247$ & 10.2 & $21.0 \pm 0$ & $6.8 \pm 0$ \\
$120 \mathrm{kVp}$ & & & & \\
DC & $150 / 241$ & 16.3 & $16.7 \pm 0.6$ & $7.3 \pm 0.2$ \\
IC & $150 / 235$ & 15.9 & $15.3 \pm 0.6$ & $8.1 \pm 0.3$ \\
\hline
\end{tabular}

Image noise values are means of three measurements with standard deviation

$D C$ discrete circuit detector, $I C$ integrated circuit detector, $C T D I_{v o l}$ volume CT dose index contrast value applying the IC detector compared with the conventional detector (Table 3). The AUC of the IC detector was significantly higher compared to the conventional detector at 80 and $100 \mathrm{kVp}(P<0.05)$ (Tables 2 and 4). No significant difference was seen for the AUC value at $120 \mathrm{kVp}$ between the two detectors $(P=0.879)$. Furthermore, there was no significant difference in the AUC between the IC detector at $80 \mathrm{kVp}$ and the conventional detector at $100 \mathrm{kVp}$ $(P=0.738)$ and between the IC detector at $100 \mathrm{kVp}$ and the conventional detector at $120 \mathrm{kVp}(P=0.506)$. Differences between readers' performance, based on comparison of the mean AUCs over all CT data sets, was not significant $(P=$ $0.835)$. The mean conspicuity of the lesions was similar at all tube voltages for both detectors ( $P=0.355-1.0$; Table 2).

Subjective evaluation of the image noise and overall image quality by the three independent readers showed a substantially higher grading for the IC detector in all tested tube voltages (Table 5). Subjective image noise and overall image quality at $100 \mathrm{kVp}$ with the IC detector was comparable to $120 \mathrm{kVp}$ with the DC detector (Fig. 2).

\section{Discussion}

Obesity and particularly its co-morbidities will be an increasing challenge for the health care systems of industrialized countries in the future. As CT is a major tool in the diagnostic work-up of secondary diseases in obese patients, the radiological society (radiologists, technicians, medical physicists and CT manufacturer) needs to be properly equipped to be able to manage the known restrictions in CT of obese patients to maintain diagnostic accuracy at lower radiation doses.

In the last few years, several technological developments were introduced to reduce the radiation dose while maintaining image quality. These technologies mostly focused on software-based modifications, such as the automatic modulation of the tube current or voltage and the reconstruction of CT images. A recent study by Desai et al. [16] showed that adaptive statistical iterative reconstruction (ASIR, GE Healthcare) substantially improves image quality in obese patients compared with filtered back projection (FBP), providing diagnostic-quality images in obese patients while reducing the radiation dose by up to $31.5 \%$ [16]. The drawback of this study was that only the first level of a health technology assessment [17], technical efficacy, was assessed. Another recent investigation on iterative reconstruction technique for obese patients (AIDR3D, Toshiba) [18] assessed not only the technical efficacy but the diagnostic effectiveness as well, which is the second level for health technology assessment. Although quantitative image quality was substantially improved by iterative reconstruction technique compared with FBP, no improvement in low-contrast detectability was 
Table 2 Data for detection of 45 simulated hypodense liver tumours from three readers

\begin{tabular}{|c|c|c|c|c|c|c|}
\hline Tube voltage and detector type & Conspicuity & No. of TP & No. of FP & Overall sensitivity (\%) & AUC & $95 \% \mathrm{CI}$ of $\mathrm{AUC}$ \\
\hline \multicolumn{7}{|l|}{$80 \mathrm{kVp}$} \\
\hline $\mathrm{DC}$ & $2.6 \pm 0.6$ & 7.7 & 10.7 & 17.2 & 0.541 & $(0.463,0.620)$ \\
\hline IC & $2.6 \pm 0.6$ & 17.0 & 10.3 & 38.1 & 0.639 & $(0.540,0.739)$ \\
\hline \multicolumn{7}{|l|}{$100 \mathrm{kVp}$} \\
\hline DC & $2.6 \pm 0.6$ & 18.3 & 18.0 & 41.0 & 0.652 & $(0.547,0.758)$ \\
\hline IC & $2.6 \pm 0.7$ & 25.7 & 11.3 & 57.0 & 0.765 & $(0.678,0.852)$ \\
\hline \multicolumn{7}{|l|}{$120 \mathrm{kVp}$} \\
\hline $\mathrm{DC}$ & $2.7 \pm 0.4$ & 28.0 & 12.0 & 62.2 & 0.790 & $(0.712,0.868)$ \\
\hline $\mathrm{IC}$ & $2.6 \pm 0.6$ & 27.3 & 7.0 & 60.7 & 0.796 & $(0.722,0.869)$ \\
\hline
\end{tabular}

Data are means of results from three independent readers. The conspicuity of the tumours was rated on a 3-point scale: 1, may be present; 2 , most likely present; 3 , definitely present

$D C$ discrete circuit detector, $I C$ integrated circuit detector, $T P$ true-positive findings, $F P$ false-positive findings, $T N$ true-negative findings, $F N$ falsenegative findings, $A U C$ area under the alternative free response operational characteristic curve

observed [18]. Thus, improved quantitative values for image quality assessment (e.g. image noise, CNR) are not necessarily accompanied by improved lesion detection. However, this important parameter should inevitably be evaluated when reducing $\mathrm{CT}$ doses.

In addition to the ambition to improve image quality at the software level, another fundamental strategy is technological innovation at the hardware level. One component of the image acquisition hardware represents the scanner's detector. In our liver phantom study, the IC detector showed a substantially lower image noise and higher CNR, resulting in a substantially improved quantitative image quality compared with the conventional detector at all three investigated tube voltages. This finding is consistent with a previously published in vitro

Table 3 Number of true-positive findings by contrast value and diameter of the simulated tumours

\begin{tabular}{|c|c|c|c|c|c|c|}
\hline \multirow[t]{2}{*}{$\begin{array}{l}\text { Tube voltage and } \\
\text { detector type }\end{array}$} & \multicolumn{3}{|c|}{$\begin{array}{l}\text { Lesion-to-liver } \\
\text { contrast (HU) }\end{array}$} & \multicolumn{3}{|c|}{ Tumour diameter (mm) } \\
\hline & 10 & 25 & 50 & 5 & 10 & 15 \\
\hline \multicolumn{7}{|l|}{$80 \mathrm{kVp}$} \\
\hline $\mathrm{DC}$ & 1.0 & 2.3 & 4.3 & 0 & 1.3 & 6.3 \\
\hline $\mathrm{IC}$ & 1.3 & 5.0 & 10.7 & 1.0 & 7.0 & 9.0 \\
\hline \multicolumn{7}{|l|}{$100 \mathrm{kVp}$} \\
\hline DC & 2.7 & 5.3 & 10.3 & 0.7 & 7.0 & 10.7 \\
\hline $\mathrm{IC}$ & 5.3 & 9.7 & 10.7 & 1.0 & 11.3 & 13.3 \\
\hline \multicolumn{7}{|l|}{$120 \mathrm{kVp}$} \\
\hline DC & 7.7 & 10.0 & 10.3 & 0.7 & 12.3 & 15.0 \\
\hline IC & 6.3 & 10.0 & 11.0 & 1.0 & 12.0 & 14.3 \\
\hline
\end{tabular}

Data were derived from reports of three independent and blinded readers. The total number of simulated tumours for each lesion-to-liver contrast value was 15 for each detector. The total number of simulated tumours for each tumour diameter was 15 for each detector

$D C$ discrete circuit detector, $I C$ integrated circuit detector and in vivo study by Morsbach et al. [19]. These authors report an increasing noise reduction with increasing phantom and patient size. The major limitations of the investigation are that only the technical efficacy and not the diagnostic effectiveness was assessed and single tube voltage of $120 \mathrm{kVp}$ was applied. In our investigation, we were able to demonstrate that the IC detector reached a significantly higher lesion detection rate of low-contrast lesions at 80 and $100 \mathrm{kVp}$ compared with conventional detector technology. The most interesting finding of our investigation is the comparable low-contrast lesion detectability between the IC detector at $100 \mathrm{kVp}$ and the conventional detector at $120 \mathrm{kVp}$. As a result, by lowering the tube voltage from 120 to $100 \mathrm{kVp}$, a radiation dose reduction of $37 \%$ can be achieved while maintaining diagnostic accuracy. Because obese patients are known to receive higher radiation doses compared with intermediate-sized patients, the IC detector has the potential to achieve substantial dose reduction, thereby improving patient safety. At $120 \mathrm{kVp}$, the IC detector did not deliver an added value in regards to the

Table 4 Comparison of performance with the various CT data sets based on the JAFROC statistic

\begin{tabular}{|c|c|c|c|c|c|c|c|}
\hline & & \multicolumn{2}{|l|}{$80 \mathrm{kVp}$} & \multicolumn{2}{|c|}{$100 \mathrm{kVp}$} & \multicolumn{2}{|c|}{$120 \mathrm{kVp}$} \\
\hline & & DC & IC & DC & IC & DC & IC \\
\hline \multirow[t]{2}{*}{$80 \mathrm{kVp}$} & $\mathrm{DC}$ & - & - & - & - & - & - \\
\hline & IC & 0.012 & - & - & - & - & - \\
\hline \multirow[t]{2}{*}{$100 \mathrm{kVp}$} & $\mathrm{DC}$ & 0.005 & 0.738 & - & - & - & - \\
\hline & IC & $<0.001$ & 0.002 & 0.004 & - & - & - \\
\hline \multirow[t]{2}{*}{$120 \mathrm{kVp}$} & $\mathrm{DC}$ & $<0.001$ & $<0.001$ & 0.001 & 0.506 & - & - \\
\hline & IC & $<0.001$ & $<0.001$ & $<0.001$ & 0.414 & 0.879 & - \\
\hline
\end{tabular}

Data are $P$ values

$D C$ discrete circuit detector, $I C$ integrated circuit detector 
Table 5 Data for subjective evaluation of image noise and image quality from three readers

\begin{tabular}{lllllllll}
\hline $\begin{array}{l}\text { Tube voltage } \\
\text { and detector type }\end{array}$ & \multicolumn{3}{l}{ Image noise } & \multicolumn{5}{c}{ Overall quality } \\
\hline $80 \mathrm{kVp}$ & $\mathrm{R} 1$ & $\mathrm{R} 2$ & $\mathrm{R} 3$ & Mean & $\mathrm{R} 1$ & $\mathrm{R} 2$ & $\mathrm{R} 3$ & Mean \\
$\mathrm{DC}$ & 1 & 1 & 1 & $1.0 \pm 0$ & 1 & 1 & 1 & $1.0 \pm 0$ \\
$\mathrm{IC}$ & 2 & 2 & 3 & $2.3 \pm 0.6$ & 3 & 3 & 2 & $2.7 \pm 0.6$ \\
$100 \mathrm{kVp}$ & & & & & & & & \\
$\quad$ DC & 2 & 2 & 2 & $2.0 \pm 0$ & 2 & 3 & 2 & $2.3 \pm 0.6$ \\
$\quad \mathrm{IC}$ & 3 & 3 & 3 & $3.0 \pm 0$ & 4 & 4 & 3 & $3.7 \pm 0.6$ \\
$120 \mathrm{kVp}$ & & & & & & & & \\
$\quad \mathrm{DC}$ & 4 & 3 & 3 & $3.3 \pm 0.6$ & 4 & 4 & 3 & $3.7 \pm 0.6$ \\
IC & 4 & 4 & 5 & $4.3 \pm 0.6$ & 4 & 4 & 4 & $4.0 \pm 0$ \\
\hline
\end{tabular}

Image noise was rated on a five-point scale (1, major, unacceptable; 2 , substantial, above average; 3 , moderate, average; 4 , minor, below average; 5 , absent). Image quality was also rated on a five-point scale (1, bad, no diagnosis possible; 2 , poor, diagnostic confidence substantially reduced; 3, moderate, but sufficient for diagnosis; 4, good; 5, excellent)

$D C$ discrete circuit detector, $I C$ integrated circuit detector

low-contrast lesion detectability and image quality compared to the conventional detector.

Radiation dose optimization in the abdomen and pelvis is particularly important because CT scans of the abdomen and pelvis are not only the most frequently performed CT studies but are also associated with highest radiation doses among all types of CT scans [20]. About half of the overall radiation exposure from CT scans is related to abdominal-pelvic CT exams [20]. The high radiation doses in the abdomen and pelvis are explained by the high attenuation of x-ray beam by the abdominal-pelvic organs. In the current study, we assessed the most challenging task in CT imaging: the

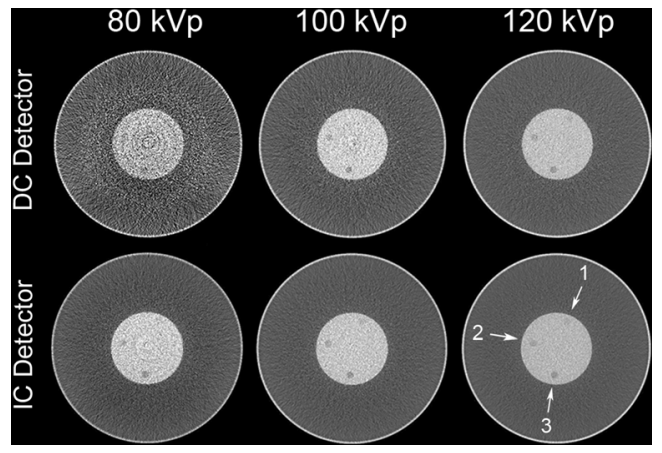

Fig. 2 Image shows the same three simulated hepatic tumours with a diameter of $15 \mathrm{~mm}$ and three different tumour-to-liver contrast values (10 HU (arrow 1), $25 \mathrm{HU}$ (arrow 2) and $50 \mathrm{HU}$ (arrow 3)) scanned with three different tube voltages $(80,100$ and $120 \mathrm{kVp})$ and two different CT scanners (equipped with an IC (bottom row) and DC detector (top row)). The same window settings are applied (window width, $400 \mathrm{HU}$; window level, $40 \mathrm{HU}$ ). The tumour with a tumour-to-liver contrast value of $10 \mathrm{HU}$ is not detected at $80 \mathrm{kVp}$ with both detectors and at $100 \mathrm{kVp}$ with the DC detector. However, this lesion is detected at $100 \mathrm{kVp}$ using the IC detector. The image noise is substantially reduced with the IC detector at all three tube voltages detection of small lesions with low contrast (5 mm lesions with tumour-to-liver contrast of $15 \mathrm{HU}$ ). However, it is important to mention that high image quality is not necessary in every abdominal-pelvic CT examination. Although tasks such as the detection of hepatic metastases in oncological imaging require high image quality, numerous clinical tasks exist in which less or reduced image quality is acceptable (e.g. ruling out renal stones, CT angiography of the abdominal aorta). Future clinical studies are necessary to assess the potential of dose optimization in obese patients for clinical tasks in which a reduced image quality is acceptable.

Recently, a general trend in CT imaging towards lowering the tube potential below $120 \mathrm{kVp}$ has occurred. The main advantages of this technique are the increase in the Hounsfield units of iodinated contrast material due to the augmented photoelectric effect and decreased Compton scattering at lower tube voltages [21]. Two recent abdominal CT investigations, a phantom study and a clinical study, confirmed this theory by demonstrating a greater Hounsfield units difference between hypovascular and hypodense lesions and hepatic parenchyma at lower tube voltages $[22,23]$. The main drawback of low tube voltages is the increased image noise caused by the reduced photon flux. To the best of our knowledge, image quality and low-contrast lesion detection have not yet been investigated at $100 \mathrm{kVp}$ in obese patients. Thus, our study is the first which demonstrates the potential use of a $100-\mathrm{kVp}$ tube voltage in obese patients undergoing abdominal CT with the IC detector. Recently, highoutput $\mathrm{x}$-ray tubes (up to $120 \mathrm{~kW}$ ) that can achieve tube current values up to $1,200 \mathrm{~mA}$ at low tube voltages have become commercially available, thus allowing obese patients to be scanned at lower tube voltages owing to a high tube current-time product.

There were some limitations of our study. First, there was a possible recall bias of the three readers in regard to the detection of the simulated tumours. However, because the reading sessions were separated by at least 1 week, the risk of a recall bias is small. Second, our liver phantom was simplified to mimic the enhancement condition of the hepatic parenchyma and hypovascular liver tumours existing momentarily during the portal venous phase. Our liver phantom, however, did not model heterogeneous parenchymal enhancement, distorted hepatic anatomy, hepatic steatosis or the different enhancing patterns of hepatic tumours. All these factors can influence the detection of hepatic tumours, especially if they are smaller than $1 \mathrm{~cm}$. Because we were interested in the relative differences of the sensitivity for lesion detection among the different protocols, the impact of the phantom's simplicity can be almost neglected. Third, our liver phantom had a cylindrical shape, which does not reflect geometry of the abdomen. Differences in upper abdomen geometry can affect the automatic tube current modulation and thus to some extent the image quality. Finally, the number of simulated liver 
lesions was rather small but large enough to find significant differences between the data sets.

In conclusion, our phantom study demonstrated the potential benefit of the novel IC detector in combination with a tube voltage of $100 \mathrm{kVp}$ for obese patients undergoing routine abdominal CT during the portal venous phase. This technical development resulted in similar subjective image quality and low-contrast detectability at a radiation dose reduction of $37 \%$ compared with a $120-\mathrm{kVp}$ protocol. Nevertheless, a future clinical study with obese patients is needed to verify if the results of our phantom study might be extrapolated into clinical practice.

Acknowledgements The scientific guarantor of this publication is Dr. Sebastian Schindera. The authors of this manuscript declare relationships with the following companies: Siemens Healthcare. This study has received funding from Siemens Healthcare. Dr. Zsolt Szucs kindly provided statistical advice for this manuscript. One of the authors has significant statistical expertise. Institutional review board approval was not required because it was a phantom study. Written informed consent was obtained from all subjects (patients) in this study. Written informed consent was waived by the institutional review board. Approval from the institutional animal care committee was obtained. Methodology: prospective, experimental, performed at one institution.

\section{References}

1. Nguyen DM, El-Serag HB (2010) The epidemiology of obesity. Gastroenterol Clin N Am 39:1-7

2. de Onis M, Blossner M, Borghi E (2010) Global prevalence and trends of overweight and obesity among preschool children. Am J Clin Nutr 92:1257-1264

3. Prospective Studies Collaboration, Whitlock G, Lewington S, Sherliker P, Clarke R et al (2009) Body-mass index and causespecific mortality in 900000 adults: collaborative analyses of 57 prospective studies. Lancet 373:1083-1096

4. Boeing H (2013) Obesity and cancer-the update 2013. Best Pract Res Clin Endocrinol Metab 27:219-227

5. Brenner DJ, Hall EJ (2007) Computed tomography-an increasing source of radiation exposure. N Engl J Med 357:2277-2284

6. Schindera ST, Nelson RC, Toth TL, Nguyen GT, Toncheva GI et al (2008) Effect of patient size on radiation dose for abdominal MDCT with automatic tube current modulation: phantom study. AJR Am J Roentgenol 190:W100-W105

7. Wang AJ, Goldsmith ZG, Wang C, Nguyen G, Astroza GM et al (2013) Obesity triples the radiation dose of stone protocol computerized tomography. J Urol 189:2142-2146

8. Sodickson A, Baeyens PF, Andriole KP, Prevedello LM, Nawfel RD et al (2009) Recurrent CT, cumulative radiation exposure, and associated radiation-induced cancer risks from CT of adults. Radiology 251:175-184

9. Smith-Bindman R, Lipson J, Marcus R, Kim KP, Mahesh M et al (2009) Radiation dose associated with common computed tomography examinations and the associated lifetime attributable risk of cancer. Arch Intern Med 169:2078-2086

10. Husarik DB, Schindera ST, Morsbach F, Chuck N, Seifert B et al (2014) Combining automated attenuation-based tube voltage selection and iterative reconstruction: a liver phantom study. Eur Radiol 24:657-667

11. Mathews JD, Forsythe AV, Brady Z, Butler MW, Goergen SK et al (2013) Cancer risk in 680,000 people exposed to computed tomography scans in childhood or adolescence: data linkage study of 11 million Australians. BMJ 346:f2360

12. Morsbach F, Desbiolles L, Plass A, Leschka S, Schmidt B et al (2013) Stenosis quantification in coronary CT angiography: impact of an integrated circuit detector with iterative reconstruction. Invest Radiol 48:32-40

13. Menke J (2005) Comparison of different body size parameters for individual dose adaptation in body CT of adults. Radiology 236:565571

14. Medicine AAoPi (2011) Size-specific dose estimates (SSDE) in pediatric and adult body CT examinations. American Association of Physicists in Medicine AAPM Report No. 204

15. Chakraborty DP (2006) Analysis of location specific observer performance data: validated extensions of the jackknife free-response (JAFROC) method. Acad Radiol 13:1187-1193

16. Desai GS, Uppot RN, Yu EW, Kambadakone AR, Sahani DV (2012) Impact of iterative reconstruction on image quality and radiation dose in multidetector CT of large body size adults. Eur Radiol 22:16311640

17. Sardanelli F, Hunink MG, Gilbert FJ, Di Leo G, Krestin GP (2010) Evidence-based radiology: why and how? Eur Radiol 20:1-15

18. Schindera ST, Odedra D, Raza SA, Kim TK, Jang HJ et al (2013) Iterative reconstruction algorithm for CT: can radiation dose be decreased while low-contrast detectability is preserved? Radiology 269:511-518

19. Morsbach F, Bickelhaupt S, Ratzer S, Schmidt B, Alkadhi H (2014) Integrated circuit detector technology in abdominal CT: added value in obese patients. AJR Am J Roentgenol 202:368-374

20. Mettler FA Jr, Bhargavan M, Faulkner K, Gilley DB, Gray JE et al (2009) Radiologic and nuclear medicine studies in the United States and worldwide: frequency, radiation dose, and comparison with other radiation sources-1950-2007. Radiology 253:520-531

21. Curry TS, Dowdey JE, Murry RC, Christensen EE (1990) Christensen's physics of diagnostic radiology, xi. Lea \& Febiger, Philadelphia, p 522

22. Schindera ST, Torrente JC, Ruder TD, Hoppe H, Marin D et al (2011) Decreased detection of hypovascular liver tumors with MDCT in obese patients: a phantom study. AJR Am J Roentgenol 196:W772W776

23. Robinson E, Babb J, Chandarana H, Macari M (2010) Dual source dual energy MDCT comparison of $80 \mathrm{kVp}$ and weighted average 120 $\mathrm{kVp}$ data for conspicuity of hypo-vascular liver metastases. Invest Radiol 45:413-418 